\title{
Imagery and contextual organization
}

\author{
IAN BEGG and DEBORAH SIKICH \\ McMaster University, Hamilton, Ontario, Canada
}

\begin{abstract}
Three experiments explore the contribution of a shared imaginal context to associative memory for pairs of items imaged interactively or separately. The major result is that an imagined context only contributes to association between items that share the context if the items are imagined as interacting with the context. Therefore, interaction effects do not reflect the contaminating influence of a shared context. Rather, context effects themselves obey the same relational principles that interaction effects in general do. The results are presented as support for the organization-redintegration hypothesis as an account of imaginal interaction.
\end{abstract}

Associative memory for pairs of words is usually far better if each pair is encoded as a single interactive image rather than as two separate images (Begg, 1973, 1978; Bower, 1970). Imaginal interaction is an effective procedure for organizing different pieces of information into new, meaningful memory units, each of which, once accessed by a cue, provides information about items related to the cue (Begg, 1982). Interaction during encoding is not the only procedure for relating items. For example, interactive imagery is not much better than separate imagery for adjective-noun pairs that are already méaningfully related (Begg, 1983); similarly, the advantage for interaction is reduced if separately imaged items are given some other basis for organization, as, for example, in imaging each item in a particular location on a clock face (Begg, 1978). Another important factor that attenuates the effect of imaginal interaction is context. Winograd and Lynn (1979) presented a series of experiments concerning the mediating role of imagined contexts in associative recall. They found that if each pair is associated with a unique context, such as a football field or a restaurant, there is not much gain in imagining items interacting in that context. That is, the context provides a strong basis for relating items, making direct interaction between them redundant.

The specific concern of this article is with the question of whether the usual advantage of interaction over separation reflects the fact that items imaged together necessarily share an imagined context or background, whereas items imaged separately do not. Perhaps a recognized cue makes contact with enough of that shared image to redintegrate the remainder, including the response item. We argue that the advantage for interaction does not reflect the confounding influence of a shared imaginal context-indeed, it is quite the reverse.

The research was funded by Grant A8122 from NSERC of Canada to Ian Begg. Experiments 2 and 3 were part of an honors thesis by the second author, supervised by the first. We thank Carolyn Jaques for her help in list preparation. Our mailing address is: Department of Psychology, McMaster University, Hamilton, Ontario L8S 4K1, Canada.
That is, a context will only be an effective mediator if the context and the to-be-related items are processed interactively. In other words, the context is a third piece of information. Imagining an item in a context is an act of interaction no different in principle from imagining any two items interacting together.

Three experiments are reported. In Experiment 1, subjects imaged the members of each pair interactively, or they imagined the members in two separate images, or they imagined the members as separate objects in a common image. The experiment allowed us to determine how much advantage shared residency in an image gives to associative recall. In Experiments 2 and 3, each pair was presented with a distinct third item that established a unique imaginal context. The members of each pair were imaged separately from each other or in interaction with each other, and, in addition, the pairs were imagined separately from or in interaction with the context word. Experiment 2 concerned associative recall of the pairs, whereas Experiment 3 concerned all the associations among the three to-be-remembered items in each set. The experiments allowed us to determine whether associative relations assessed by recall measures are isomorphic with imaginal interactions established during initial encoding.

\section{EXPERIMENT 1}

Experiment 1 asked whether shared residency in a single image is sufficient to remove any advantage of interaction between items. Three study procedures were contrasted. One procedure was separate imagery. Subjects formed a single image for each of the 120 words in a 60-pair list. Another procedure was interactive imagery. Subjects formed a single image for each pair, with the two members interacting in that image. Previous contrasts between the two procedures have shown marked differences in recall, on the order of $60 \%$ as opposed to $10 \%$ (Begg, 1978). The third condition, the sharedimage condition, was a blend of the other two. As in the interaction condition, subjects formed a single image for each pair, but as in the separation condition, the 
members were to be kept separate from each other within that image. Bower $(1970,1972)$ contrasted separation of this type with interaction and found an advantage for interaction. However, the three conditions are necessary to determine how much advantage over total separation is provided by sharing an image, and how much additional advantage is provided by interaction within that image.

According to the organization-redintegration hypothesis (Begg, 1982), the interaction condition should yield relatively good cued recall because recognition of the cue is relatively likely to make available a unitary trace of the appropriate pair, including the response member. Separation should be poor because the recognized cue accesses a trace that contains no information about the required response. In the shared-image condition, performance should exceed separation because the trace contacted by a recognized cue contains information beyond the cue itself, so that there is some likelihood that the imaged background will allow redintegration of the entire image, including the response. It does not follow as a deduction from the organization-redintegration hypothesis that interaction should exceed the shared-image condition. If there were some way to measure the extent to which each imaginal background is unitary, the hypothesis would predict that highly unitary backgrounds would lead to performance similar to interaction, and that highly fragmentary backgrounds, with the pair members in different fragments, would approximate separation. Since for the shared-image condition to reach interaction it would require that all the images were unitary, it is most likely that the sharedimage condition falls between the other two.

The experiment also included a test of item recognition. According to the organization-redintegration hypothesis, interaction specifically affects the degree of organization or relation among items. Recognition depends not on organization but, rather, on item discriminability. Interaction does not, in and of itself, have any consequence for discriminability, and, in general, interactive and separate imagery do not differ in item recognition (Begg, 1978, 1982; Dempster \& Rohwer, 1974; Bower, 1970). If interactively imaging an item produces enough context-specificity to make the image different from a more usual encoding, then interaction may hurt recognition, as has been found in a few studies (Begg, 1979, 1983; McGee, 1980). However, the point is that item discriminability, as reflected in recognition, and organization, as reflected in associative recall, are independent attributes of a memory trace. Under the present range of parametric variation, there is no reason to expect differential item recognition. In other words, the conditions should differ in the organization of the memory system, not in the amount of information retained.

\section{Method}

Subjects. Fifty-four introductory psychology students from
McMaster University served as a course requirement, in three groups of 18.

Materials. A total of 180 nouns with I $>5.99$ were selected from Paivio, Yuille, and Madigan (1968). The nouns were sorted at random to form 60 pairs, leaving 60 nouns to be distractors in a later recognition test. The 60 pairs were videotaped at the rate of $5 \mathrm{sec}$ per pair. A recognition test was constructed with the 60 distractors and one member of each studied pair; half of these had been the right-hand member during study. A cuedrecall test was also constructed, using one member from each pair; half of these cues had been targets in recognition, and half had been right-hand members during study.

Procedure. In each of the three experiments, the subjects were told initially that the purpose of the experiment was to compare the effectiveness of different ways of studying material for a later memory test of unspecified type. They were encouraged to use the procedure to be described to them, even if they felt they could do better on their own. Also in each of the three experiments, following the description of the particular encoding procedure, all subjects were instructed specifically to keep "each image separate from each other image, so you don't get them confused later" (see Begg, 1978).

The subjects studied the pairs in one of three ways. In the interactive condition, the subjects formed a single interactive image for each pair, in an imagined frame above the monitor. In the separate condition, the subjects imaged the left-hand member of each pair in an imagined frame to the left of the monitor, and the right-hand member to the right of the monitor. In the shared-image condition, the subjects imaged both members in an imagined frame above the monitor, but kept the two separate within that image. Following study, the subjects completed the recognition test and then the recall test, with $7 \mathrm{~min}$ allowed for each test. In each phase, they were encouraged to circle or write down all the words they could remember.

\section{Results and Discussion}

The retention measures appear in Table 1, with the three study tasks in columns and several measures of retention in rows. It is clear that the study tasks were equivalent in all measures of recognition. Thus, different imaginal organizations do not necessarily lead to differential discriminability of the imagined items. It is also clear from Table 1 that the conditions were very different in recall $[F(2,51)=33.7]$. Interactive imagery led to good recall $(.53)$, whereas separate imagery yielded poor recall $(.10)$. The interesting condition is the sharedimage condition, for which the recall level (.31) was intermediate between those of the other two conditions. Thus, having a shared imaginal context for a pair of

Table 1

Retention Measures After Three Study Tasks

\begin{tabular}{|c|c|c|c|c|c|c|}
\hline & \multicolumn{6}{|c|}{ Study Task } \\
\hline & \multicolumn{2}{|c|}{ Interactive } & \multicolumn{2}{|c|}{ Shared } & \multicolumn{2}{|c|}{ Separate } \\
\hline & Mean & SD & Mean & SD & Mean & SD \\
\hline \multicolumn{7}{|l|}{ Measure } \\
\hline False Alarms & .08 & .08 & .07 & .08 & .07 & .07 \\
\hline Recognition Hits & .84 & .08 & .86 & .09 & .86 & .12 \\
\hline Mean Recall & .53 & .21 & .31 & .12 & .10 & .13 \\
\hline \multicolumn{7}{|l|}{ Recall After: } \\
\hline Cue as Target & .48 & .21 & .28 & .10 & .06 & .09 \\
\hline Response as Target & .58 & .21 & .34 & .16 & .14 & .17 \\
\hline
\end{tabular}


nouns leads to better associative recall than does total separation, as found by Winograd and Lynn (1979). However, associative recall mediated by a shared image is not as good as associative recall mediated by direct interaction between the cue and the response. Table 1 also shows a contrast between pairs whose cue was the recognition target and those whose response was the target. The extra study occasion afforded by the recognition test clearly benefits recall more if the response is the item receiving the extra study $[\mathrm{F}(1,51)=16.3]$; this effect is typical (see Begg, 1979).

Perhaps the most important result is that the factors affecting associative recall are independent of the factors affecting item discrimination, even if the tasks are conducted successively on the same subjects. Interactive imagery is not sufficient to change discriminability of individual items relative to separate imagery. Allowing separately imaged items to share an imaginal context does not affect discrimination, but it does increase the level of association between the items, presumably because each item is associated with the same context. Clearly, however, such sharing is not sufficient to produce as strong a relation between items as is direct interaction.

\section{EXPERIMENT 2}

In Experiment 1, context was manipulated by asking the subjects to place separately imaged items in one image, rather than in two. The addition of the shared context to separately imaged items was beneficial for associative recall, but did not produce levels of recall as high as those produced by direct interaction between the cue and the response. In Experiment 2, context was manipulated by providing a unique third item for each pair. Winograd and Lynn (1979) provided locations (e.g., restaurant) as contexts. However, it seems, intuitively, to be difficult to imagine an item separately from a location presented at the same time. Since the intent of the experiment was to contrast items imagined separately from or in interaction with the context, we decided to use as contexts either the names of classes of objects (e.g., tool, musician, animal) or specific exemplars of those classes (e.g., hammer, pianist, horse).

As stated above, the concern of the experiment was with whether the presence or absence of a shared imaginal context affects the advantage of interactively imaged pairs over separately imaged pairs. The subjects studied 30 pairs of nouns either by imagining the two members, $\mathbf{A}$ and $\mathbf{B}$, interacting with each other or separately from each other. Crossed with pair interaction was context interaction. Each pair appeared with a unique third item, $\mathrm{C}$, the context for the pair. Interactively imaged pairs were imagined either interacting with or separately from their context. Similarly, each member of separately imaged pairs was imagined interacting with or separately from its context. The retention measure was recall of $B$ with $A$ as a cue.
It seems clear that recall of $B$ should be better with A as the cue if $A$ and $B$ are interactively imaged, since the items are members of the same memory trace, than if $A$ and $B$ are separately imaged, since the items are members of different traces (Begg, 1982). The addition of a shared context should not help in the case of interactive images, since the interaction already provides a basis for relating the items. However, if the pair members are separately imaged, then the addition of a shared context should be of benefit, since the context can provide a basis for relating the items in memory: A is interacting with $\mathrm{C}$, and $\mathrm{C}$ is interacting with $\mathrm{B}$. Consequently, the advantage for pair interaction over pair separation should be smaller if the pair members share a context than if they do not share a context.

Recall that, above, the contexts were either relatively general (e.g., tool) or relatively specific (e.g., hammer). It is reasonable to expect that more specific terms are more recognizable than more general terms (e.g., Reder, Anderson, \& Bjork, 1974), presumably because their encodings are relatively constant over occasions. Consequently, specific context should be especially advantageous for items imagined separately from each other but in interaction with the context. Thus, the trace for $A$ and $C$ should be better able to contact the trace for $\mathrm{C}$ and $\mathrm{B}$ if $\mathrm{C}$ is specific rather than general, in which case the two representations of $\mathrm{C}$ might differ in significant ways. An equivalent expectation is that the advantage of interactive over separate imagery for pairs should be smallest in the case in which items share a specific context. If so, the locations used by Winograd and Lynn (1979) as contexts could represent a limiting case of the specificity of the context, and hence be the least fertile ground for an advantage of interaction over separation as procedures for representing pairs.

\section{Method}

Subjects. The subjects were 160 introductory psychology students from McMaster University, who fulfilled a course requirement by their participation. Ten students were assigned to each of 16 conditions.

Materials. The materials consisted of 30 pairs of to-beremembered words and 30 pairs of context words. The to-beremembered words were selected from Paivio et al. (1968), with $\mathrm{I}>5.99$ and with $\mathrm{F} \geqslant \mathrm{A}$ for half and $\mathrm{F}<\mathrm{A}$ for the other half. The context words were also chosen from Paivio et al. Thirty were relatively general categorical terms, such as animal, musician, and tool; mean $\mathrm{I}=6.10$, and $\mathrm{F} \geqslant \mathrm{A}$ for half the words. The other half were specific exemplars of the categories, such as horse, pianist, and hammer; mean $I=6.26$, and $F \geqslant A$ for half the words.

Two different lists were prepared. The lists were videotaped at the rate of one trial every $10 \mathrm{sec}$; the context word appeared at the top of the screen, and the pair members appeared side by side beneath it. The general context words appeared in one list, and their more specific exemplars appeared in the other.

Two different test lists were prepared, one with the 30 lefthand items and one with the 30 left-hand items and context words as cues.

Procedure. As in the other experiments, the subjects were first encouraged to do their best to comply with the specific instructions that would follow, and, after having heard the 
specific instructions, they were told specifically to keep each image separate from each other image. The subjects studied the list under one of four imagery conditions resulting from a factorial combination of pair imagery (interactive or separate) and context imagery (interactive or separate). In the interactiveinteractive condition, the subjects formed a single image of the three items interacting and projected the image above the monitor. In the interactive-separate condition, the subjects projected an image of the context word above the monitor and an image of the pair members interacting below the monitor. In separation-separation, a single image of each of the three words was projected to a different side of the monitor. Finally, in separation-interaction, the subjects projected an image of the lefthand item and context interacting to the left and then an image of the right-hand item and context to the right. Subjects in all conditions were told that the top word in each triplet was the context and that the bottom words were a pair, and the terms "context," "pair," and "member of the pair" were used throughout the instructions.

Following study, the subjects attempted to recall the righthand items, given either the left-hand items as cues or the lefthand items and context words as cues, with $5 \mathrm{~min}$ allowed for completion. The subjects were encouraged to recall as many of the target words as they could during the recall period.

\section{Results and Discussion}

The main recall values were analyzed by a $2 \times 2 \times 2 \times$ 2 between-subjects analysis of variance, with context (general or specific), cues (item or item plus context), contextual imagery (interactive or separate), and pair imagery (interactive or separate) as the factors. Since the different cuing conditions entered in no reliable effects, the results are averaged over that variable for presentation in Table 2 . The only reliable main effect was that of pair interaction $[\mathrm{F}(1,144)=59.4]$, with interactive imagery, $.40(\mathrm{SD}=.27)$, exceeding separate imagery, $.14(.16)$. Contextual imagery interacted with pair imagery $[F(1,144)=7.07]$, as seen by the values for the means in Table 2 . Interaction with context helped recall of separately imaged pairs, but not of interactively imaged pairs.

Table 2 shows the results separately for the general and the specific contexts, since the three-way interaction was reliable $[F(1,144)=4.51]$. As expected, the smallest advantage for pair interaction over pair separation

Table 2

Recall of Interactively and Separately Imaged Pairs, Imaged Either Separately From or In Interaction With Specific or General Contexts

\begin{tabular}{lcc}
\multicolumn{1}{c}{ Pairs } & $\begin{array}{c}\text { Interactive with } \\
\text { Context }\end{array}$ & $\begin{array}{c}\text { Separate from } \\
\text { Context }\end{array}$ \\
\hline Interactive & General Context & \\
Separate & .41 & .35 \\
& .16 & .06 \\
Interactive & Specific Context & .49 \\
Separate & .35 & .07 \\
& .25 & .42 \\
Interactive & Mean & .06 \\
\hline Separate & .38 & \\
\hline
\end{tabular}

Note-The MSe value for the above means is .047 . occurred if the pairs were interacting with a specific context (.35 vs. .25). It was also the case that the largest advantage for contextual interaction over contextual separation occurred with separately imaged pairs associated with a specific context (.25 vs. .07$)$, although there was also an advantage for separately imaged pairs associated with a general context (.16 vs. .06). Adding a shared context to interactive pairs may have helped slightly if the context was general (.41 vs. .35), but if the context was specific, its addition hurt substantially (.35 vs. .49$)$.

That context interaction helps in remembering separately imaged pairs accords well with the organizationredintegration hypothesis. That context interaction is of no great value for pairs that are already interactive also is no surprise, since the additional interaction is redundant, and may even be harmful in some cases. The hypothesis neither predicts nor is compromised by the finding that, with specific contexts, context interaction hurts recall of interactively imaged pairs. Perhaps there is some reduction in pairwise relational information if subjects attempt to form an interactive image of three, rather than two, items, if one of those items is very specific. However, regardless of the explanation, the negative effect of contextual interaction was interesting enough for further study.

\section{EXPERIMENT 3}

Experiment 3 used the specific-context list from Experiment 2 and investigated interitem associations more directly. Recall was cued by either the A term or the $\mathrm{C}$ term, in each case with the remaining two items as responses. The basic expectation was simply that the organization shown by associations in recall tracks the interactions imaginally encoded during study. Thus, for totally interactive triplets, all associations among A, B, and $C$ should be strong and lead to high levels of recall and interitem association. In contrast, total separation should lead to low levels on all measures. The more challenging cases are the remaining two. If the $\mathrm{AB}$ pair is interactive, but separate from the context, then the only case with good recall should be that with $A$ as the cue and $B$ as the response, and the only case with a high association between to-be-remembered items should be that with $\mathrm{C}$ as the cue; $\mathrm{C}$ should not be a good cue, but whenever either A or B is recalled, the other should follow. Finally, with A and B separately associated with $\mathrm{C}$, there should be strong $\mathrm{AC}$ and $\mathrm{BC}$ associations, but no direct $A B$ association. Thus, recall of $C$ and recall cued by $C$ should be especially good, with recall of $B$ cued by A being somewhat worse, and measures of association should generally be relatively high, except that the $\mathrm{AB}$ association should be weaker than the rest.

\section{Method}

Subjects. Eight groups of 20 subjects were made up of intro- 
ductory psychology students who participated to meet a course requirement.

Materials and Procedure. The study list of 30 pairs with specific contexts, videotaped at a 10 -sec rate, was retained from Experiment 2. The subjects studied the list by using one of the four imagery conditions resulting from factorially crossing instructions for pair interaction or separation with instructions for context interaction or separation, also as in Experiment 2. Following study, the subjects were given either the $30 \mathrm{~A}$ terms or the $30 \mathrm{C}$ terms as cues on a prepared sheet, with two spaces, for the other two members, adjacent to each cue. They were encouraged to write the two missing words, in either order, next to each cue.

\section{Results and Discussion}

Recall was analyzed in several ways. First, consider the number of items recalled. Table 3 presents mean recall proportions for the four study conditions for the two items remaining for each cue. The results were anlayzed by a 2 (pair interaction vs. separation) $\times 2$ (context interaction vs. separation) $\mathrm{x} 2$ ( $\mathrm{A}$ as the cue or $\mathrm{C}$ as the cue) $\mathrm{x} 2$ ( $\mathrm{B}$ as the response or the other term as the response) analysis of variance, with the last factor being a within-subjects factor. Since 12 of the $15 \mathrm{~F}$ ratios were reliable, the results are interpreted by post hoc contrasts, based on a $t$ value calculated from the meansquare error $(.0151)$ for the four-way interaction $[F(1,152)=6.87]$; any two means that differ by .08 are reliably different at $\alpha=.05$.

There are several ways of contrasting the means. First, consider the four rows. The first two rows show a strong advantage for context interaction, but no advantage for pair interaction. These results are exactly as expected, since all the measures depend on associations with $\mathrm{C}$, the context. The third row, which also depends on associations with $\mathrm{C}$, likewise shows a strong advantage for context interaction over context separation. Additionally, pair separation is actually better than pair interaction; perhaps the $\mathrm{AC}$ association is stronger if it is a specifically formed interaction rather than part of a larger one. However, the three rows tell much the same story; if recall requires associative information linking an item to a context, then performance is much better if the associations were encoded as interactive images than if the items were separately imaged.

The fourth row is very different from the other three, and replicates the results of Experiment 2. Since

Table 3

Proportion of Items Recalled, Depending on Imagery and Cue Conditions

Imagery Condition

\begin{tabular}{|c|c|c|c|c|c|c|}
\hline \multirow[b]{2}{*}{ (Cue) } & \multirow{2}{*}{\multicolumn{2}{|c|}{$\rightarrow$ Response }} & \multicolumn{2}{|c|}{ Context Interactive } & \multicolumn{2}{|c|}{ Context Separate } \\
\hline & & & PI & PS & PI & PS \\
\hline (C) & $\rightarrow$ & A & .50 & .47 & .06 & .06 \\
\hline (C) & $\rightarrow$ & B & .46 & .40 & .06 & .05 \\
\hline (A) & $\rightarrow$ & $\mathrm{C}$ & .40 & .50 & .04 & .06 \\
\hline (A) & $\rightarrow$ & B & .36 & .25 & .64 & .06 \\
\hline
\end{tabular}

Note-MSe $=.0151 ;$ a difference between means of .08 is reliable by a post hoc $t$ test. $P I=$ pair interactive; $P S=$ pair separate .
Table 4

Measures of Association in Experiment 3

\begin{tabular}{|c|c|c|c|c|c|c|}
\hline \multirow[b]{3}{*}{ (Cue) } & \multirow[b]{3}{*}{$\rightarrow$} & \multirow[b]{3}{*}{ Pair } & \multicolumn{4}{|c|}{ Imagery Condition } \\
\hline & & & \multicolumn{2}{|c|}{ Context Interactive } & \multicolumn{2}{|c|}{ Context Separate } \\
\hline & & & PI & PS & PI & PS \\
\hline \multicolumn{7}{|c|}{ Pair Recall } \\
\hline (A) & $\rightarrow$ & $\mathrm{BC}$ & .28 & .24 & .03 & .02 \\
\hline (C) & $\rightarrow$ & $\mathrm{AB}$ & .40 & .25 & .06 & .03 \\
\hline \multicolumn{7}{|c|}{ Conditional Recall } \\
\hline (A) & $\rightarrow$ & $\mathrm{BC}$ & .67 & .51 & .09 & .28 \\
\hline (C) & $\rightarrow$ & $\mathbf{A B}$ & .78 & .49 & .94 & .29 \\
\hline
\end{tabular}

Note-MSe for pairs $=.027 ;$ MSe for conditional probabilities $=$ .063. $P I=$ pair interactive; $P S=$ pair separate.

performance depends on the $A B$ association, pair interaction exceeds pair separation. Context interaction helps only if the pair members are separate, in which case the shared context provides a mediational link to the response. If the pair is already interactive, then the addition of the context appears to hurt, replicating the negative effect of context found in Experiment 1.

The conditions allow eight contrasts of pair interaction versus pair separation. Interaction helps only in the two contrasts in which the $A B$ association is directly assessed (.36 vs. .25 , and .64 vs. .06). There are also eight contrasts for context interaction versus separation. Interaction is very helpful for the six contrasts in which the context item is either the cue or the response. With $\mathrm{A}$ as the cue for $\mathrm{B}$, context interaction helps (.25 vs. .06) if the paired items are separately imaged from each other and hurts (.36 vs. .64) if the pair members are interactive.

Associative measures. Table 4 presents the proportion of pairs recalled and the proportion of recalled words that were correctly paired. In the analysis of the number of pairs recalled, there was a large main effect of context interaction $[F(1,152)=102]$ and a smaller, but reliable, effect of pair interaction $[F(1,152)=4.83]$. However, six of the seven $F$ ratios were reliable with conditional measures, with the three-way interaction of $F(1,152)=19.9$ and a post hoc critical difference of .16. The degree of association in recall was high (.67 and $.78)$ if all three items were interacting, moderate (.51 and .49) if the A and B items were separately interactive with the context, and low (.28 and .29) if all three items were separate. If the pair was interactive but separate from the context, then the $A B$ association was exceptionally strong (.94) and the $\mathrm{BC}$ association exceptionally weak (.09). Thus, although the interactive pairs separate from the context were rarely recalled with the context as the cue, what little recall there was consisted almost entirely of pairs; if A was the cue, however, there was much more recall, but that recall consisted almost entirely of B items in isolation.

Finally, Table 5 presents the breakdown of each condition into all possible recall events. Following Winograd and Lynn (1979), a Fisher exact probability 
Table 5

Partition of Recall Even ts in Experiment 3

\begin{tabular}{|c|c|c|c|c|c|c|c|c|c|c|}
\hline \multirow[b]{2}{*}{ Interaction } & \multicolumn{5}{|c|}{$A$ as the Cue } & \multicolumn{5}{|c|}{$\mathrm{C}$ as the Cue } \\
\hline & $B$ and $C$ & B only & C only & Neither & $\mathrm{Z}$ & $A$ and $B$ & A only & B only & Neither & $\mathrm{Z}$ \\
\hline Pair and Context & .28 & .08 & .12 & .52 & -10.0 & .40 & .10 & .06 & .44 & -12.4 \\
\hline Context Only & .24 & .01 & .26 & .49 & -6.14 & .25 & .21 & .15 & .39 & -3.54 \\
\hline Pair Oniy & .03 & .61 & .01 & .36 & +7.28 & .06 & .00 & .00 & .94 & -5.11 \\
\hline Neither & .02 & .04 & .03 & .91 & +4.43 & .03 & .03 & .02 & .92 & +6.53 \\
\hline
\end{tabular}

was calculated for each subject's $2 \times 2$ matrix, the probability was converted to a $Z$ score, and the sum of the $\mathrm{Z}$ scores for each condition was divided by $\mathrm{N}^{1 / 2}$, providing a test of whether the departure from independence was reliable. A reliable departure is signaled by a negative $Z$ score; positive $Z$ scores mean merely that the obtained distribution is not at all unlikely, given the null hypothesis of independence.

The cases of interaction between the pair members and the context show nearly all-or-none recall, with only about .18 of the cases resulting in recall of single items; these two conditions show strong dependency in recall. The cases with the pair members separately interacting with the context also showed reliable dependency in recall, but less than with total interaction. More single items were recalled in these conditions, although, with $A$ as the cue, subjects almost never (.01) recalled $\mathrm{B}$ without also recalling $\mathrm{C}$. The pair-only cases differ from each other. With $A$ as the cue, subjects tended to recall $B$ or nothing, producing a pattern that does not show dependency between $B$ and $C$. However, with $C$ as the cue, recall was none-or-all (especially none), showing reliable dependency between $\mathrm{A}$ and $\mathrm{B}$. Finally, in the total separation conditions, the subjects tended to recall nothing or fragments, showing no departure from independence.

\section{Conclusion}

The results clearly showed that the organization of recall reflects interaction during study, regardless of whether the interaction is between paired items or items and a unique context. Interaction with a context can increase recall of items that are not themselves imaged together, and can thereby greatly attenuate the effectiveness of direct interaction between pair members. However, there is some evidence that an image of a pairwise interaction is more effective as a means of remembering that pair than is an image of which that pair is a part. Apparently, there is some degree of tradeoff, whereby the addition of more information increases the range over which the image provides information but, in so doing, reduces the amount of relational information that pertains to particular associations.

\section{GENERAL DISCUSSION}

All three experiments examined the relationship between initial encoding and the degree to which items are associated in recall. A facile, but not inaccurate, summary is "you get out of memory what you put into it." If two items are encoded as a single interactive image, then associative recall of the items is relatively high, whether assessed by recall of one cued by the other or by the conditional probability that the two items will appear together rather than separately, given some other item as a cue. According to the organizationredintegration hypothesis (Begg, 1982), the relationship between associative recall and interactive study reflects both encoding and retrieval processes. Interactive study increases the likelihood that each pair will be encoded as a single memory unit that, once accessed by a cue, is redintegrated, thereby making available information that can be decoded as the desired response. Redintegration will not provide response information if the response was encoded separately from the cue, with the result that items processed separately lead to relatively poor levels of associative memory performance.

By the account, it matters little whether an item is presented as a member of a pair or as a unique context for the pair. In either event, redintegration during retrieval, resulting in relatively high levels of associative recall, will only occur if the items between which the association is required were processed interactively. However, the degree to which direct interaction between two items exceeds separately processed items is attenuated if the two separately processed items are both processed interactively with a third item. The degree of attenuation is especially great if that third item is specific rather than general, presumably because the specific context is likely to be invariant in the two traces, enabling one, once it is redintegrated, to access the other.

The results are in good accord with the organizationredintegration hypothesis. The results are also similar to those reported by Winograd and Lynn (1979), except that their concern was with the difference between unique and shared contexts, rather than with that between interactive and separate contexts. Since they used locations as contexts, it seems reasonable to assume that items were encoded interactively with the contexts. Thus, their conclusion that the "provision of an imaginary context is regarded as a sufficient condition for establishing A-B organization" (p. 34) is comparable to the encoding assumption of the organization-redintegration hypothesis. Likewise, their discussion of encoding uniqueness (cf. Lesgold \& Goldman, 1973) would lead 
one to expect greater advantage for specific than for general items as context for otherwise separately studied pairs, as was found here. That is, we see no disagreement between the results and account of Winograd and Lynn and the present results and account. Winograd and Lynn's locations were especially potent mediators, but that difference between the two sets of experiments is not a matter of principle as much as of degree. Perhaps locations such as restaurants, cars, and football fields are especially specific contexts if they are imagined (see Anderson, Pichert, Goetz, Schallert, Stevens, \& Trollip, 1976). Alternatively, perhaps the procedure they used, in which both items were encoded in the context, is especially conducive to interaction, relative to the present procedure, in which the separate items were separately interactive with the context.

By the present account, context is functionally an additional piece of information that can be interactively processed with other items, and that can later mediate associative retrieval among otherwise unrelated items. Thus, context takes its place among other factors that reduce the need for, and hence reduce the value of, direct association between items. For example, meaningful pairs do not show much benefit if they are imagined in interaction rather than separately (Begg, 1983). Similarly, children remember noun pairs better if the nouns are linked by verbs rather than by conjunctions. However, the advantage for verbs disappears if the pairs are studied either interactively or separately, since interaction is of more benefit for conjunction-linked nouns than for verb-linked nouns, whereas separation hurts memory for verb-linked nouns more than it does that for conjunction-linked nouns (Begg \& Young, 1977). Thus, imaginal interaction is not the only procedure for relating items, and other means of relating items sometimes make interaction redundant.

Petersen (1974) used a different approach to the question of contextual mediation and found some interesting results. His subjects studied noun triplets interactively with each other and with a context. The manipulation of interest was that one member of the triplet, the member that was later to be the recall cue, was either of high or low imagery value and was either strongly or weakly associated with the context. For example, with the context WEDDING, the triplet consisted of "dress" and "ring" and one of four possible cues, namely "bride" (concrete, highly associated with WEDDING), "candle" (concrete, less associated with WEDDING), "vows" (abstract, highly associated), or "beauty" (abstract, less associated). Recall of the two words "dress" and "ring" depended on the degree of association between the cue and the context, but not on the concreteness of the cues. Of course, ratings of imagery in isolation are not identical to ratings in specific contexts (Begg \& Clark, 1975), so that the lack of an effect of concreteness is difficult to interpret. However, the fact that cues highly associated with the context lead to better recall than do less associated cues fits well with the expectation that interaction during study between an item and a context is necessary for the item later to access the context, enabling redintegration of the other responses.

None of the results discussed to this point requires any modification of the organization-redintegration hypothesis, beyond stating the tacit possibility that a third item can enter into interactive organization with other items. However, several results do point to the acknowledgement that the value of an interactive image decreases as more items are incorporated. Thus, whereas, in principle, it does not matter how many items are represented in an image, in practice, there appears to be a tradeoff in which gains in the bandwidth, the number of items to which an image pertains, are offset to some degree by a loss in fidelity, the precision with which any particular item or relationship is encoded by that image (see McGee, 1980). This interesting problem deserves further study in its own right, particularly since context can, under some circumstances, hurt associative recall.

Finally, it may be interesting to contrast the present mediational account with traditional mediational accounts, exemplified by Kjeldergaard (1968), as they apply to the shared-context condition. First, the accounts differ in their characterization of encoding. By the present account, there are two traces: The interactive image of $A$ and $C$ contains information pertinent to $A$, to $C$, and to the relationship between them, whereas the image of $\mathrm{C}$ and $\mathrm{B}$ contains information pertinent to $C, B$, and their relationship. By Kjeldergaard's account, since $A, B$, and $C$ are familiar words, each will be responded to as a whole, so that what is learned is an association between $A$ as a stimulus and $B$ as a response, and an association between $B$ as a stimulus and $\mathrm{C}$ as a response. Retrieval in Kjeldergaard's account is a sequence in which the stimulus $\mathbf{A}$, responded to as a whole, elicits the response $B$, which in turn elicits the response $\mathbf{C}$; thus, the elicitation of $\mathbf{C}$ by $\mathbf{A}$ is a multiplicative function of the momentary strengths of the A-C and C-B habits (assumption 23, p. 72). In the present account, the cue $\mathrm{A}$ as encoded at test makes contact with the pertinent subset of the $\mathrm{AC}$ trace, at which time the trace is redintegrated, making its informational contents available. If the information pertinent to $\mathrm{C}$ thus accessed is sufficiently similar to the information pertinent to $C$ in the $C B$ trace, that trace is accessed and redintegrated, allowing recall of $\mathrm{B}$. Clearly, either account is compatible with the present results. The primary difference lies in the conception of encoding; the older account characterizes encoding of familiar units as being invariant over occasions and contexts, except insofar as the set of covert associations may differ, whereas the present account characterizes encoding as a relatively efficient process in which trace contents are determined by the discriminative needs of the task. Interestingly, the account by Kjeldergaard does consider the integration of nonsense syllables as stimuli 
and responses to be sensitive to task demands, with different elements encoded under different conditions. Words, however, were considered as familiar and invariant wholes by most theorists in the 1960s, with the current conceptions of contextual variability (e.g., Light \& Carter-Sobell, 1970) and encoding specificity (e.g, Tulving \& Thomson, 1973) emerging somewhat later. In principle, we see no reason why an account such as Kjeldergaard's would be compromised by allowing more variability in word encoding, but we doubt that contemporary writers will recast their interpretations in terms of S-R habits between samples of elements.

\section{REFERENCES}

Anderson, R. C., Pichert, J. W., Goetz, E. T., Schallert, D. L., Stevens, K. V., \& Trollip, S. R. (1976). Instantiation of general terms. Journal of Verbal Learning and Verbal Behavior, 15, 667-679.

BEGG, I. (1973). Imagery and integration in the recall of words. Canadian Journal of Psychology, 27, 159-167.

BEGG, I. (1978). Imagery and organization in memory: Instructional effects. Memory \& Cognition, 6, 174-183.

BegG, I. (1979). Trace loss and the recognition failure of unrecalled words. Memory \& Cognition, 7, 113-123.

BEGG, I. (1982). Imagery, organization, and discriminative processes. Canadian Journal of Psychology, 36, 273-290.

BEGG, I. (1983). Imagery instructions and the organization of memory. In J. C. Yuille (Ed.), Imagery, memory and cognition. Hillsdale, NJ: Erlbaum.

BegG, I., \& ClarK, J. M. (1975). Contextual imagery in meaning and memory. Memory \& Cognition, 3, 117-122.

BEGG, I., \& Young, B. J. (1977). An organizational analysis of the form class effect. Journal of Experimental Child Psychology, 23, 503-519.
Bower, G. H. (1970). Imagery as a relational organizer in associative learning. Journal of Verbal Learning and Verbal Behavior, 9, 529.533.

BowER, G. H. (1972). Mental imagery and associative learning. In L. W. Gregg (Ed.), Cognition in learning and memory. New York: Wiley.

Dempster, R. N., \& Rohwer, W. D. (1974). Component analysis of the elaborative encoding effect in children's learning. Journal of Experimental Psychology, 103, 400-408.

KJeldergaARD, P. M. (1968). Transfer and mediation in verbal learning. In T. R. Dixon \& D. L. Horton (Eds.), Verbal behavior and general behavior theory (pp. 67-96). Englewood Cliffs, NJ: Prentice-Hall.

Lesgold, A. R., \& Goldman, S. R. (1973). Encoding uniqueness and the imagery mnemonic is associative learing. Journal of Verbal Learning and Verbal Behavior, 12, 193-202.

Light, L. L., \& Carter-Sobell, L. (1970). Effect of changed semantic context on recognition memory. Journal of Verbal Learning and Verbal Behavior, 9, 1-11.

MCGEe, R. (1980). Imagery and recognition memory: The effects of relational organization. Memory \& Cognition, 8, 394-399.

Paivio, A., Yuille, J. C., \& Madioan, S. A. (1968). Concreteness, imagery, and meaningfulness values for 925 nouns. Journal of Experimental Psychology Monographs, 76(1, Pt. 2).

Petensen, R. C. (1974). Imagery and cued recall: Concreteness or context? Journal of Experimental Psychology, 102, 841-844.

Reder, L. M., Anderson, J. R., \& Bjonk, R. A. (1974). A semantic interpretation of encoding specificity. Journal of Experimental Psychology, 102, 648-656.

Tulving, E., \& Thomson, D. M. (1973). Encoding specificity and retrieval processes in episodic memory. Psychological Review, 80, 352-373.

Winograd, E., \& Lynn, D. A. (1979). Role of contextual imagery in associative recall. Memory \& Cognition, 7, 29-34.

(Manuscript received June 10, 1983; revision accepted for publication September 27, 1983.) 